\title{
Estimation of welding distortions and straightening workload trough a data mining analysis*
}

\author{
Losseau Nicolas ${ }^{1,3, a}$, Caprace Jean David ${ }^{1,4, b}$, Archambeau Dominique ${ }^{2, c}$, \\ Amrane Amirouche ${ }^{1, d}$, Rigo Philippe ${ }^{1,4, e}$ \\ ${ }^{I}$ University of Liège, Chemin des chevreuils B52, 4000 Liege - Belgium \\ ${ }^{2}$ PEPITe ; Rue des Chasseurs Ardennais, 4031 Liège, Belgium \\ ${ }^{3}$ Fund for Training in Research in Industry and Agriculture of Belgium (F.R.I.A.) \\ ${ }^{4}$ National Fund of Scientific Research of Belgium (F.N.R.S.) \\ ${ }^{a}$ n.losseau@ulg.ac.be, ${ }^{b}$ jd.caprace@ulg.ac.be, ${ }^{c}$ d.archambeau@pepite.be, \\ da.amrane@ulg.ac.be, ${ }^{\mathrm{e}}$ ph.rigo@ulg.ac.be
}

Keywords: welding distortions, straightening, shipbuilding, cost assessment, data mining

Abstract

This paper will present a way to minimize cost in shipbuilding industry by using the results of a data mining analysis aiming to improve the cost knowledge of the straightening process.

This statistical analysis was based on production data from a shipyard and has the scope to establish an assessment formula of the straightening workload. An intermediate step of the analysis was to estimate the welding distortions appearing in stiffened panels. Those generated formulas are useful to improve the research in the following domains: production simulation, cost assessment of ship hull, structure optimization, design for production, etc.

\section{Introduction}

Since several years, the big shipyards use more and more plates of small thickness to build up the stiffened panels in order to decrease the structural weight of ships. The major problem relating to the utilization of thin plates is the appearance of welding distortions that have to be eliminated for esthetical and service reasons. This straightening operation involves non negligible costs and it seems thus important to characterize its economical impact on the global hull fabrication.

The idea used to establish the straightening workload formulation was to lead a statistical analysis basing on the production data from a shipyard. This paper gathers the results of two complementary analyses. The first analysis has exploited workload data of 13 passengers' ships and has ensured to establish a relation linking the scantling (geometrical characteristics of stiffened panels) to the straightening cost [hour $\left./ \mathrm{m}^{2}\right]$. The second analysis was recently led further to a measure campaign gathering the welding distortions of one cruise ship; this data mining study exploited

\footnotetext{
* A part of this paper results from part of the work performed in sub-project II.1 of InterSHIP, a European R\&D project funded under the European Commission's Sixth Framework Programme for Research and Technological Development. (Project nº TIP3-CT-2004-506127)
}

Losseau, Straightening Estimation, 1 
those previous data in order to estimate the distortions and to improve the straightening assessment formula.
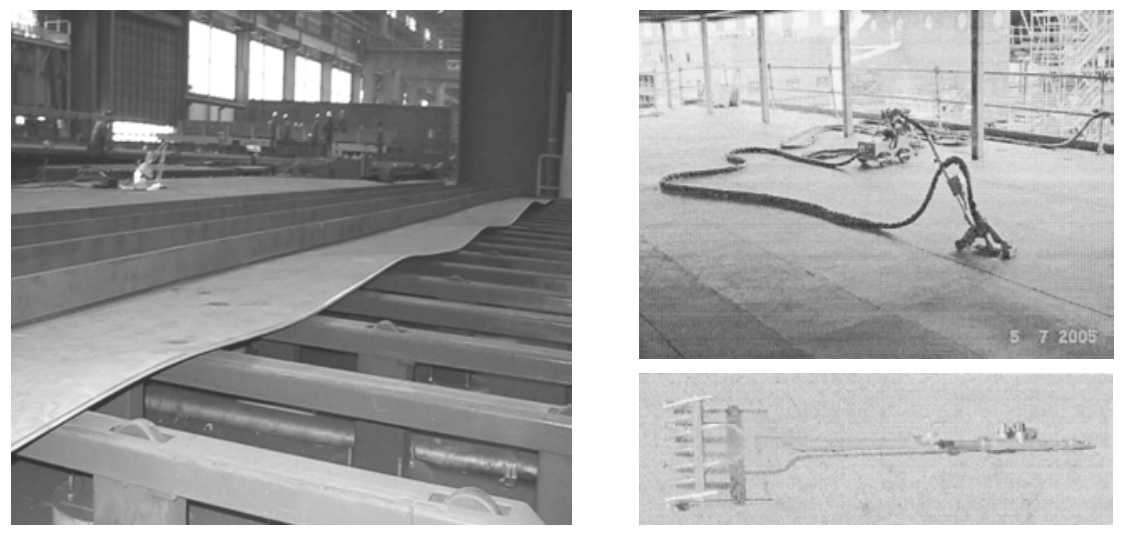

Figure 1 (1) A distorted panel.

Straightening techniques: (2) by induction, (3) with a 5 nozzles blowtorch.

This paper summarises each stage of the data mining methodology: data feeding, data quality analysis, data exploration, choice of discriminatory attributes and finally generation of the formulae. Some interesting techniques coming from Pepito $\subset$ software were used in order to perform this analysis like: linear correlations analysis by dendrogram tools, conditioned histograms, conditioned dots clouds, decision tree tools (based on minimisation entropy) and a Neuronal Network Analysis.
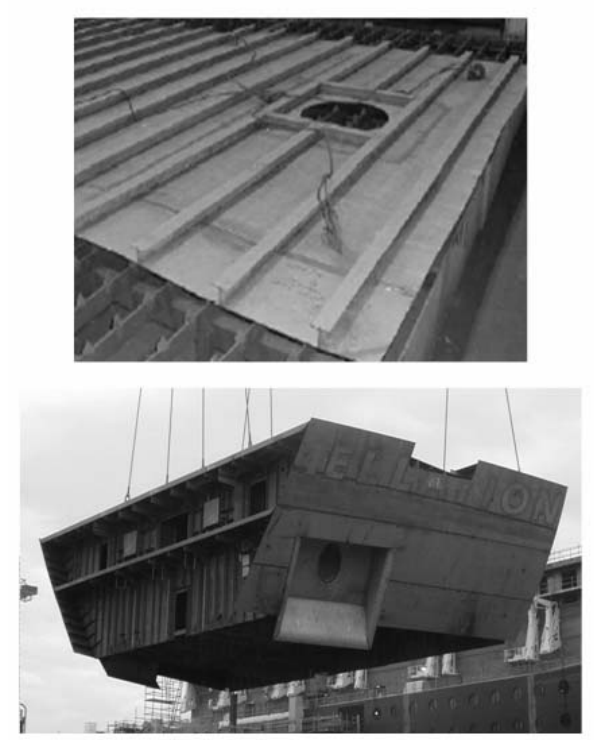
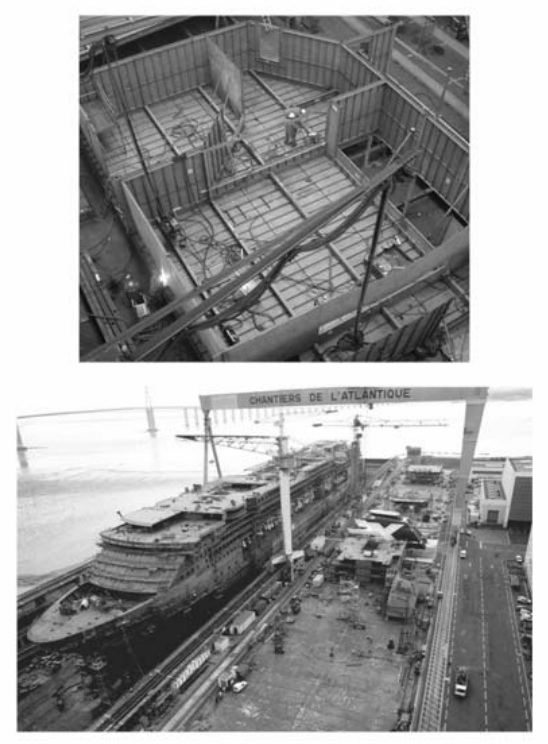

Figure 2 : (1) panel, (2) section, (3) block, (4) pre-mounting zone and dry dock

Losseau, Straightening Estimation, 2 


\section{DFE2008 Design, Fabrication and Economy of Welded Structures}

\section{Fabrication methodology in shipbuilding}

The shipbuilding industry utilises the concept of modular fabrication; the ship is divided in assemblies and sub-assemblies and those elements are fabricated in specialised workshops in order to reach a high productivity.

The basic elements called panels $\left(\sim 15 \mathrm{~m}^{*} 15 \mathrm{~m}\right)$ are constituted by side plates and longitudinal stiffeners. Then transversal girders and stiffened partitions are assembled on panels to realise sections $(\sim 15 \mathrm{~m} * 30 \mathrm{~m} * 3 \mathrm{~m})$; the simple geometric sections are fabricated in production lines while complex ones are built in hall assemblies. The next stage (pre-mounting work) is the realisation of blocks $\left(\sim 30 \mathrm{~m}^{*} 30 \mathrm{~m} * 12 \mathrm{~m}\right)$ by assembling several sections. Finally, the blocks are transported in the dry dock and connected together to constitute the ship. The straightening operations are done in the dry dock by experimented workers.

\section{Straightening costs assessment: first analysis}

The analysis of straightening assessment has followed the Data Mining methodology that is, by definition, the non-trivial process of extracting valid, previously unknown, comprehensible, and useful information from large databases. The successive steps of the analysis were the following ones:

\subsection{Database creation}

A data base was first of all constituted; it gathered, for 13 passenger's ships, the characteristics of each section (global geometry, deck thickness, dimensions and inter-distance of stiffeners, section family, deck number, steel grade, section weight, etc.) and the associated straightening workload [hour].

\subsection{Data description stage}

This step consisted in a presentation of the attributes (fields of the data base), with their distribution and other statistical parameters (minimum, maximum, mean and variance).

One of difficulties which arose during the database analysis is that the most structural attributes show a discrete distribution with one or few dominant modes (see Figure 3 (3) and (4)); for instance, the distance between stiffeners has very often the same value. Those attributes are almost "constant" parameters and thus don't constitute a conclusive information source. In order to minimize this effect, we have replaced some attributes. In this scope, we divided the plate weight by the section surface to obtain information similar to the thickness, but having the advantage to present a distribution much less discrete.

\subsection{Data quality stage}

This step listed the problematic recordings (strange distribution, missed values, data in conflict with their physical meaning) in order to take care of them in the next stages. 


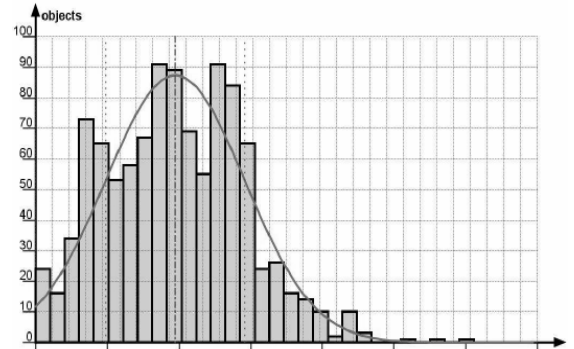

(1) Sections Surface

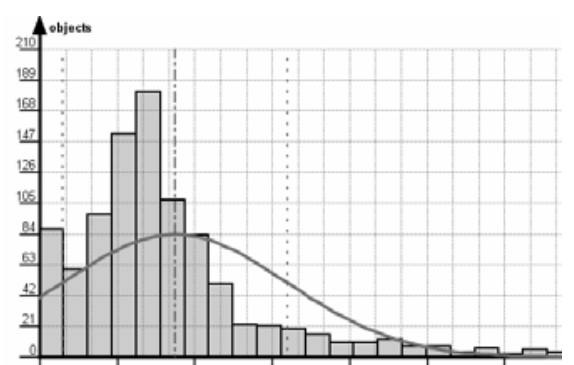

(2) Straightening time

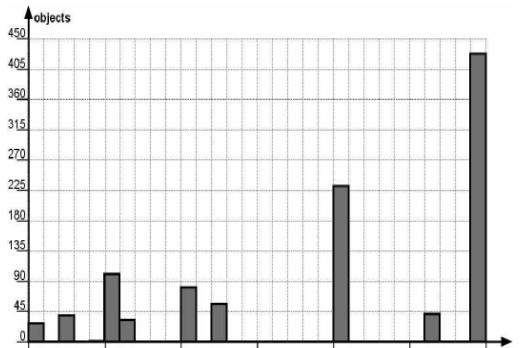

(3) Distrance between stiffeners

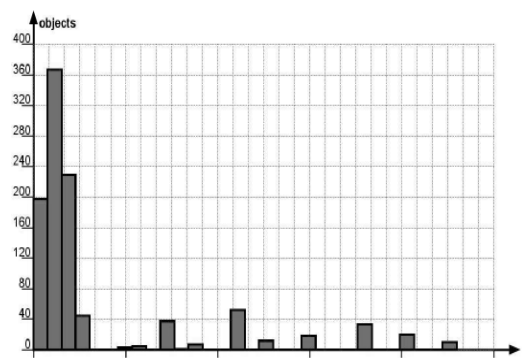

(4) Thickness

Figure 3. Distribution histograms of attributes

A particular point has been noticed at this stage; the values related to the straightening work realised by sub-contractors are not reliable since this workload corresponds to an estimated time and not a time of strictly effectuated work. Unhopefully this case concerns more than two third of the records and decreases thus the quantity of exploitable data.
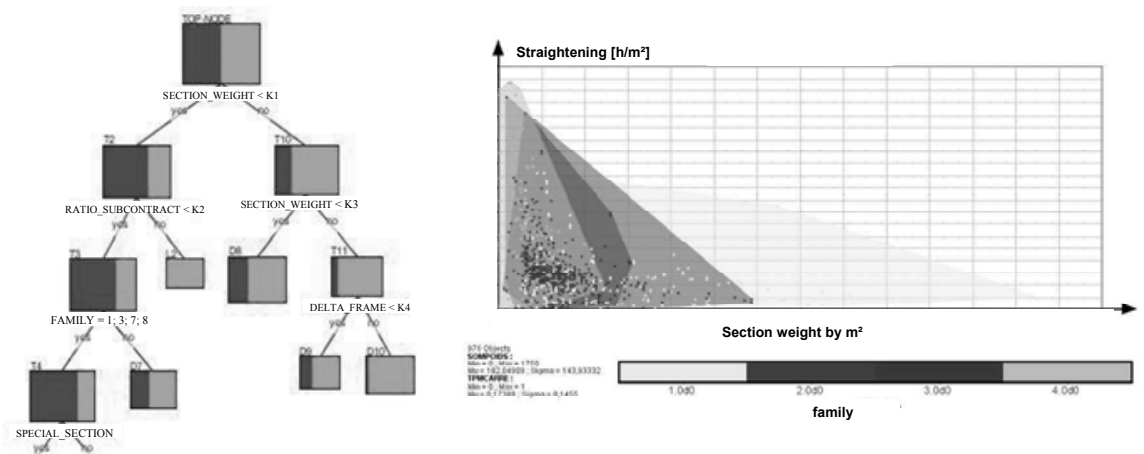

Figure 4. Statistical tools: (1) decision tree, (2) conditioned dots clouds

\subsection{Data exploration stage}

This work stage consisted in using different approaches to visualize the correlations existing between the attributes and the straightening workload in order to finally select the parameters having the most relevant influence on the straightening assessment. In order to fulfil this stage, four different approaches were used: a linear 


\section{DFE2008 Design, Fabrication and Economy of Welded Structures}

correlation analysis trough dendrograms elaboration, conditioned histograms, conventional dots clouds diagrams and decision trees analyses.

\subsection{Elaboration of the formula}

This stage consisted in building the relation between the straightening cost [hour $\left./ \mathrm{m}^{2}\right]$ and the sections characteristics. The technique selected was the Artificial Neural Networks (ANN) method who is a powerful technique permitting to elaborate non linear relations (i.e. hyperbolic tangents) linking several inputs to a unique output.

The input attributes selected to generate the formula were the following ones: thickness, longitudinal stiffeners spacing, transversal girders spacing, ratio stiffeners spacing/girders spacing, section family, section weight $/ \mathrm{m}^{2}$, section weight $/$ section length. After having chosen the input parameters, it was necessary to restrict the number of records in order to ignore the sections carrying perturbing information. In this optic, we have ignored the sections whose the straightening work was done by sub-contractors because time measurements of straightening were less reliable.

The formula was elaborated exploiting 273 records and the correlation between the real value of straightening cost and the value estimated by the formula was 0,838 .
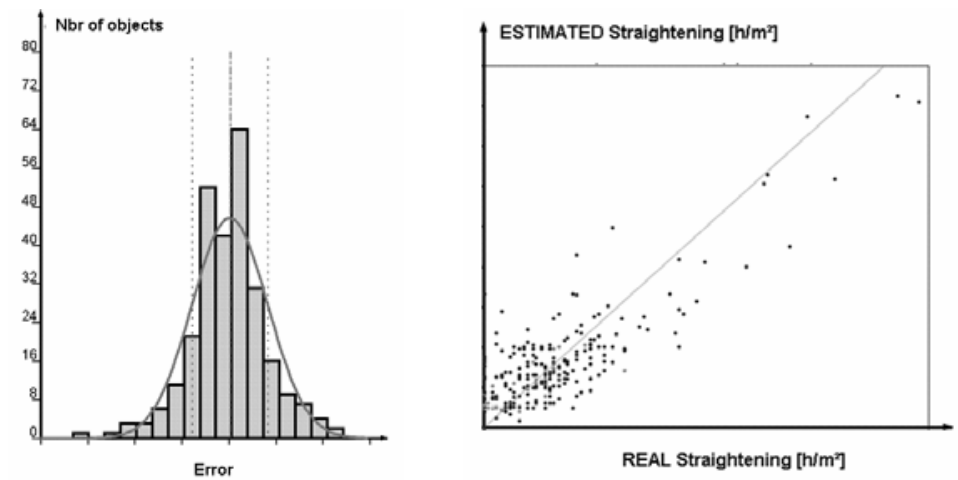

Figure 5 : errors diagrams relating to straightening work estimation (from PEPITo ${ }^{\circledR}$ )

\subsection{Limitation of the formula}

We have to notice that the generated formula has a limit. Firstly, since the recordings were restricted to the works realised by the shipyard workers, the quantity of data exploited was small and thus the robustness of the formula was not excellent. Moreover, when we have constructed the error diagrams, we have voluntarily tested the equation on the same data set than the one used to establish the relation. A consequence of this choice is that the precision given is not representative. Those precisions are optimist in comparison of the precision obtained if the test set was different than the learning set.

\section{4. distortions assessment}

\section{1 data description}


Further to a measure campaign gathering the deck distortions of one passenger ship, the data mining analysis relating to welding distortions and straightening has been revived. The deformations were measured after the blocks assembly stage and before the straightening operations. Each recorded value was related to the distortion occurring at the middle of rectangles (about $0,7 \mathrm{~m} * 2,5 \mathrm{~m}$ ) delimited by consecutive longitudinal stiffeners and consecutive transversal girders. The campaign covered about $50 \%$ of the total deck surface and was associated only to small thicknesses from 5 to $8 \mathrm{~mm}$.

Those distortions correspond thus to the accumulation of welding deformations occurring at each fabrication stage. During the sections fabrication, the welding of plates and longitudinal stiffeners generate firstly a system of bulges that is then modified and sometimes accentuated during the fixing of the transversal girders and bulkheads. The fabrication stage realising the connection of sections to constitute blocks involves important traction efforts on the plate extremities and modifies again the distortions system. Finally, the resulting decks deformations vary in configuration and in direction; there can be one or several bulges between two consecutive girders and the value of distortions can be positive or negative. Nevertheless, in $70 \%$ of the cases, the plates are distorted in the side of the stiffeners and only one bulge is present between successive girders. An example of deformations field is presented in the Figure 6; colours have been applied in order to try to visualise characteristics of the distortions system and eventual correlations between data.

\subsection{Data mining stages}

The database gathers for each rectangle delimited by consecutive stiffeners and girders: plate thickness, stiffeners spacing, girders spacing, steel grade, presence of a plate junction, coordinates of the rectangle inside the ship, deck number, etc. A particular attribute called "additional process" describes the utilisation of a distortion reduction technique (such as application of weld seams onto the plate between stiffeners). Indeed the initial scope of the measure campaign was to evaluate the impact of such distortions reduction techniques.

We added other attributes about the section: section weight by $\mathrm{m}^{2}$, section workshop ( 0 for assembly halls; 1 or 2 respectively for the production line of the partially automated workshop). We generate also combined parameters: distortion/thickness in order to have attributes without units.

The successive data mining stages were then applied to: investigate the correlation between attributes, extract meaningful parameters, fix exploitable data sub-sets and finally elaborate a formula trough an ANN analysis.

\section{3 establishment of the formula}

Numerous attempts have been done to generate an effective estimation formula. It has been revealed that the exploitation of each distortion measure taken as unique record generates too much noise and involves thus a poor quality estimation (correlation around 0,5 for 23000 elements). By gathering the data by plates (1400 
elements) the correlation increased to 0,7 and by gathering the data by sections (108 elements) the correlation reached 0,9 . This last solution was thus utilised to estimate the welding distortion in absolute value.
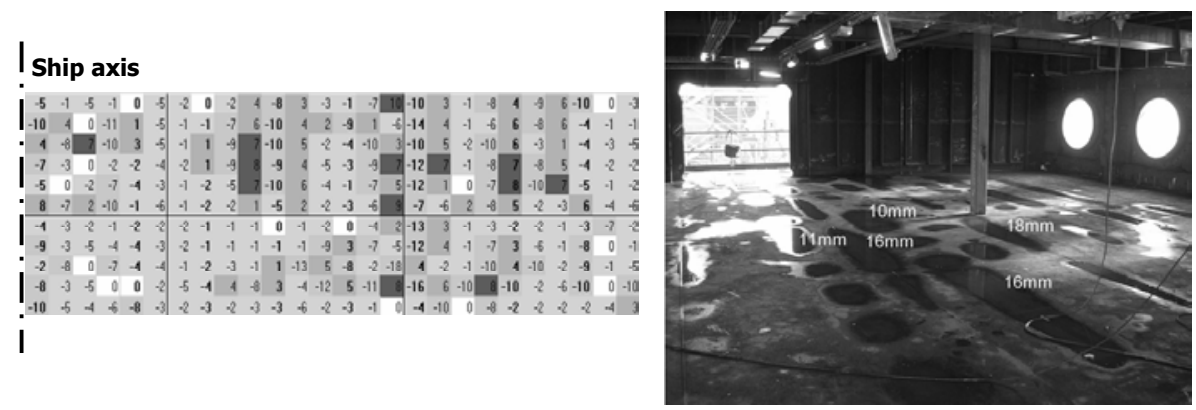

Figure 6 : (1) visualisation of a distortions field, (2) photo of a distorted deck

To generate the formula, the 108 sections concerned by the measured campaign were utilised and the following attributes were introduced as inputs: plate thickness, longitudinal stiffeners spacing, transversal girders spacing, ratio stiffeners spacing/girders spacing, section weight by $\mathrm{m}^{2}$, workshop of the section, additional process.

The correlation between the real distortions and the values estimated by the formula was 0,947 (see Figure 7). This value is optimist because the data set to test the formula was the same than the set to generate the formula.
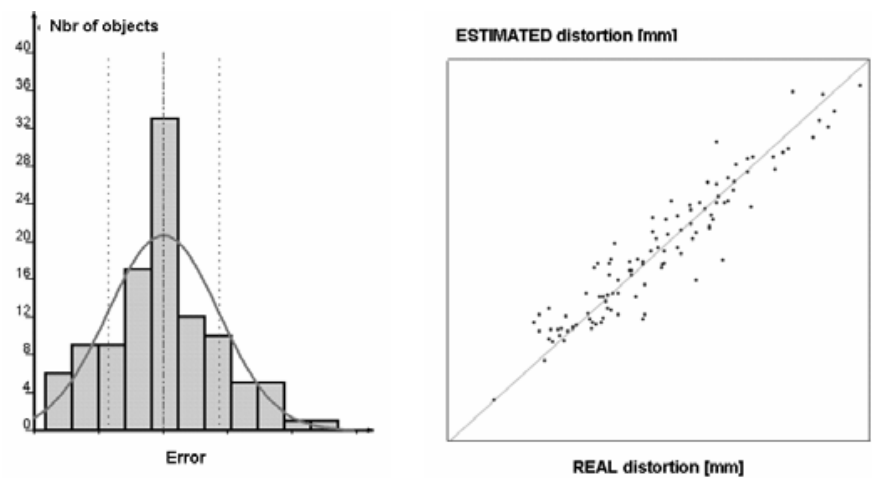

Figure 7 : errors diagrams relating to welding distortions estimation (from PEPITo ${ }^{\circledR}$ )

Here again, the formula has a limitation. The estimation is quite good inside the range values encountered by the attributes in the database but is not excellent outside. For instance, an estimation relating to a thickness lower than $5 \mathrm{~mm}$ or higher $8 \mathrm{~mm}$ becomes rapidly approximate.

\section{5. straightening assessment : second analysis}

Losseau, Straightening Estimation, 7 
The knowledge of the distortions values seemed interesting to exploit in the straightening assessment. In effect, intuitively, higher are the distortions, longer will be the straightening operations.

The first idea was thus to generate a straightening estimation formula using the real distortions and the real straightening workload of the ship concerned by the measure campaign. Unhopefully, the straightening works of this ship being totally effectuated by sub-contractors, the data were thus not reliable. The second idea was then to introduce the estimated distortions as a supplementary input into the straightening formula in order to improve the prediction. The Artificial Neural Networks technique was thus launched with this new parameter; this permitted to increase the formula correlation from 0,838 to 0,862 .

\section{Conclusions}

The basic structures used in shipbuilding are stiffened panels that are assembled by welded joints. The welding operations generate distortions that accumulate along the fabrication stages and that have to be eliminated for esthetical and service reasons. The common utilisation of thin plates increases the straightening operations and it seems thus important to characterise those supplementary works that involve over costs and delivery delays.

This paper summarises two data mining analyses that exploited production data from the Saint Nazaire shipyard. The first study permitted, trough a statistical analysis of 13 ships data, to establish a relation linking the straightening workload to the sections characteristics. The second one aimed to estimate the welding distortions from the scantling data. A step complementary to the first analysis was then realised; the distortions estimated by the formula were introduced as a supplementary input into the straightening equation.

Those generated formulas are useful to improve the research in the following domains: production simulation, cost assessment of ship hull, structure optimization, design for production, etc. Moreover, the advantages of the distortion formula are the rapidity and the simplicity that are very important points in industry. Indeed, it permits to evaluate rapidly the value of welding distortions without using more complex methods such Finite Elements.

\section{Acknowledgments}

The authors thank University of Liege, AKERYARD FRANCE (Saint-Nazaire) and Pepite (Liège), for the collaboration within sub-project II.1 of InterSHIP.

\section{References}

Caprace JD., Losseau N., Archambeau D. (2007) A Data Mining Analysis Applied to a Straightening Process Database. In: International Conference COMPIT 2007 pp. 186-197

Chau T.T., Jancart F. and Bechepay G. (2001) About the welding effects on thin stiffened panel assemblies in shipbuilding. In: International Conference on Marine Technology Odra 2001, Szczecin, Poland.

Bruce G. \& Morgan G. (2006) Artificial Neuronal Networks - Application to freight rates. In: International Conference COMPIT 2006, pp. 146-154

Losseau, Straightening Estimation, 8 